\title{
Gaussian field theory for the Brownian motion of a solvated particle
}

\author{
Thomas Speck ${ }^{1}$ \\ ${ }^{1}$ Institut für Theoretische Physik II, Heinrich-Heine-Universität Düsseldorf, \\ Universitätsstraße 1, 40225 Düsseldorf, Germany
}

\begin{abstract}
An alternative derivation of Brownian motion is presented. Instead of supplementing the linearized Navier-Stokes equation with a fluctuating force, we directly assume a Gaussian action functional for solvent velocity fluctuations. Solvating a particle amounts to expelling the solvent and prescribing a boundary condition to the solvent on the interface that is shared with the solute. We study the dynamical effects of this boundary condition on the solvent and derive explicit expressions for the solvent mean flow and velocity correlations. Moreover, we show that the probability to observe solvent velocity fluctuations that are compatible with the boundary condition reproduces random Brownian motion of the solvated particle. We explicitly calculate the translational and rotational diffusion coefficients of a spherical particle using the presented formalism.
\end{abstract}

PACS numbers: 05.40.-a,05.40.Jc

\section{INTRODUCTION}

Our traditional understanding of Brownian motion is that of a solute getting kicked randomly by surrounding solvent molecules. Einstein's seminal contribution had been to derive the diffusion equation-and in particular an explicit expression for the diffusion coefficient-based on this insight [1. One route to derive Brownian motion from more fundamental equations is fluctuating hydrodynamics 2, which augments the linearized NavierStokes equations with a random stress tensor obeying the fluctuation-dissipation theorem. Integrating out the fluid velocity field, the stochastic equation of motion for a solvated particle can then be derived, which takes on the form of a generalized Langevin equation [3. This approach is not without problems since the correlations of the random stress tensor near the solute are assumed to be the same as those in the isotropic bulk fluid. There is, however, evidence that the presence of rigid bodies influences the local stress fluctuations [4].

The purpose of this Brief Report is to give a somewhat alternative view on the dynamics of solvated particles, a view in which Brownian motion arises from suppressing solvent fluctuations. Instead of the stress, we consider directly the (memoryless) fluctuations of the solvent velocity. We employ Gaussian field theory [5] to study how a solute modifies these velocity fluctuations in its vicinity. Gaussian field theory has been applied successfully to, e.g., dielectric relaxation dynamics [6] and the hydrophobic effect [7. In analogy to the modification of density fluctuations due to the excluded volume of the solute, we show that imposing a solvent velocity on the solute-solvent interface leads to a flow and diminishes solvent velocity fluctuations in the vicinity of the solute. Loosely speaking, the solvent loses entropy, which is compensated by the random motion of the solute.

\section{THE PURE SOLVENT}

We consider an incompressible quiescent fluid with bulk viscosity $\eta$. We assume that in the absence of any solvated object the probability $P_{0}[\mathbf{u}]=\exp \left\{-\mathcal{S}_{0}[\mathbf{u}]\right\}$ to observe a given history of velocity fluctuations $\mathbf{u}(\mathbf{r}, t)$ away from zero is Gaussian with action

$$
\mathcal{S}_{0}[\mathbf{u}]=\frac{1}{2} \int \mathrm{d} t \int \mathrm{d}^{3} \mathbf{r d}^{3} \mathbf{r}^{\prime} \mathbf{u}(\mathbf{r}, t) \cdot \boldsymbol{\chi}_{0}^{-1}\left(\mathbf{r}, \mathbf{r}^{\prime}\right) \cdot \mathbf{u}\left(\mathbf{r}^{\prime}, t\right) .
$$

The functional inverse of $\chi_{0}$ is defined through

$$
\int \mathrm{d}^{3} \mathbf{r}^{\prime \prime} \chi_{0}^{-1}\left(\mathbf{r}, \mathbf{r}^{\prime \prime}\right) \chi_{0}\left(\mathbf{r}^{\prime \prime}, \mathbf{r}^{\prime}\right)=\mathbf{1} \delta\left(\mathbf{r}-\mathbf{r}^{\prime}\right),
$$

where 1 denotes the identity matrix. Normalization of $P_{0}$ is achieved through choosing the appropiate functional measure $[\mathrm{d} \mathbf{u}]$. Clearly, the matrix $\chi_{0}$ is related to the velocity correlations,

$$
\left\langle\mathbf{u}(\mathbf{r}, t) \mathbf{u}^{T}\left(\mathbf{r}^{\prime}, t^{\prime}\right)\right\rangle_{0}=\chi_{0}\left(\mathbf{r}, \mathbf{r}^{\prime}\right) \delta\left(t-t^{\prime}\right) .
$$

The brackets denote averages over different realizations of the velocity field, whereas the subscript indicates the pure solvent. Eq. (3) implies temporally uncorrelated velocity fluctuations. We know that this is not strictly correct as hydrodynamics predicts a power law tail for the particle velocity autocorrelation function [8, 9]. However, for the sake of simplicity, here we aim for a simple Markovian description which is appropriate for sufficiently coarse-grained time. Brownian motion in confined geometries such as nanopores [10] might require to take into account memory.

Now image that an external force density $\mathbf{f}(\mathbf{r}, t)$ acts on the solvent. Up to linear order, the fluctuationdissipation theorem [11] relates this force to an instantaneous mean velocity profile

$$
\langle\mathbf{u}(\mathbf{r}, t)\rangle=\frac{1}{2 T} \int \mathrm{d}^{3} \mathbf{r}^{\prime} \chi_{0}\left(\mathbf{r}, \mathbf{r}^{\prime}\right) \cdot \mathbf{f}\left(\mathbf{r}^{\prime}, t\right)
$$

through the correlations Eq. (3). The factor $1 / 2$ originates from the time integration over the $\delta$-function. Of 
course, Eq. 4 is nothing else than Faxen's theorem, from which we can deduce the correlations

$$
\chi_{0}(\mathbf{r})=\frac{T}{4 \pi \eta r}\left(\mathbf{1}+\frac{\mathbf{r r}^{T}}{r^{2}}\right)
$$

using the Oseen tensor 12. However, we will not need an explicit expression for $\chi_{0}$ in the following.

\section{SOLVATING A PARTICLE}

We solvate a particle expelling the fluid from the volume $V$ the particle occupies. For the clarity of presentation, we consider a spherical particle. Then rotational and translational diffusion decouple and in the following we focus on the translational diffusion. The particle is described by its instantaneous velocity v. By choosing the velocity correlations Eq. (3) we assume a time scale separation between the relaxation time of the fluid and the time scale on which the solvated particle diffuses. From the perspective of the solute it thus appears as if the solvent responds instantaneously with a mean flow profile $\langle\mathbf{u}(\mathbf{r})\rangle_{\mathbf{v}}$ to a change of $\mathbf{v}$.

To calculate this flow profile, we exploit the time scale separation through fixing $\mathbf{v}$ and consider the generating function

$$
Q[\phi] \equiv \int[\mathrm{d} \mathbf{u}] \int[\mathrm{d} \boldsymbol{\psi}] e^{-\mathcal{S}_{0}+\mathrm{i} C+\int \mathrm{d} t \int \mathrm{d}^{3} \mathbf{r} \phi(\mathbf{r}, t) \cdot \mathbf{u}(\mathbf{r}, t)}
$$

with conjugate field $\phi(\mathbf{r}, t)$. We implement the "no-slip" or "stick" boundary condition such that the fluid layer on the particle surface has the same velocity as the moving particle. The boundary condition gives rise to the constraint

$$
\prod_{t} \delta(\mathbf{u}(\mathbf{r}, t)-\mathbf{v}(t))=\int[\mathrm{d} \boldsymbol{\psi}] e^{\mathrm{i} C[\boldsymbol{\psi}]}
$$

in Eq. (6) with functional

$$
C[\boldsymbol{\psi}, \mathbf{u} \mid \mathbf{v}] \equiv \int \mathrm{d} t \int \mathrm{d}^{3} \mathbf{r}[\mathbf{u}(\mathbf{r}, t)-\mathbf{v}(t)] \cdot \boldsymbol{\psi}(\mathbf{r}, t)
$$

The auxiliary function $\boldsymbol{\psi}(\mathbf{r}, t)$ is non-zero only on the particle surface $\delta V$ and zero elsewhere.

We evaluate the path integrals in Eq. 6) in two steps. First, integration over the velocity fluctuations results in

$$
Q[\phi]=\int[\mathrm{d} \boldsymbol{\psi}] \exp \left\{-\frac{1}{2} \int \mathrm{d} t \boldsymbol{\psi} \circ \boldsymbol{\chi}_{\mathrm{s}} \circ \boldsymbol{\psi}+\mathrm{i} \int \mathrm{d} t \boldsymbol{\psi} \circ \mathbf{b}+\frac{1}{2} \int \mathrm{d} t \int \mathrm{d}^{3} \mathbf{r d}^{3} \mathbf{r}^{\prime} \boldsymbol{\phi}(\mathbf{r}, t) \cdot \boldsymbol{\chi}_{0}\left(\mathbf{r}, \mathbf{r}^{\prime}\right) \cdot \boldsymbol{\phi}\left(\mathbf{r}^{\prime}, t\right)\right\}
$$

To ease the notational burden, we define the operation

$$
\mathbf{g} \circ \mathbf{h} \equiv \int_{\delta V} \mathrm{~d}^{2} \mathbf{r} \mathbf{g}^{T}(\mathbf{r}) \mathbf{h}(\mathbf{r})
$$

as the integral over all positions that are elements of the particle surface. The matrix $\chi_{\mathrm{s}}$ quantifies fluid velocity correlations in the absence of, but on the interface the fluid would share with, the solvated particle. The linear term in Eq. (8) couples to the vector

$$
\mathbf{b}(\mathbf{r}, t) \equiv \int \mathrm{d}^{3} \mathbf{r}^{\prime} \chi_{0}\left(\mathbf{r}, \mathbf{r}^{\prime}\right) \cdot \phi\left(\mathbf{r}^{\prime}, t\right)-\mathbf{v}(t)
$$

where $\mathbf{r} \in \delta V$. Performing the integration over the auxiliary vector field $\boldsymbol{\psi}$ we finally obtain the generating function

$$
\begin{aligned}
& Q[\phi]=\mathcal{N} \exp \left\{-\frac{1}{2} \int \mathrm{d} t \mathbf{b} \circ \chi_{\mathrm{s}}^{-1} \circ \mathbf{b}\right. \\
& \left.+\frac{1}{2} \int \mathrm{d} t \int \mathrm{d}^{3} \mathbf{r} \mathrm{d}^{3} \mathbf{r}^{\prime} \boldsymbol{\phi}(\mathbf{r}, t) \cdot \chi_{0}\left(\mathbf{r}, \mathbf{r}^{\prime}\right) \cdot \boldsymbol{\phi}\left(\mathbf{r}^{\prime}, t\right)\right\}
\end{aligned}
$$

with (irrelevant) prefactor $\mathcal{N}$ due to the functional determinant of $\chi_{\mathrm{s}}$. The inverse matrix is determined through

$$
\chi_{\mathrm{s}}\left(\mathbf{r}, \mathbf{r}^{\prime \prime}\right) \circ \chi_{\mathrm{s}}^{-1}\left(\mathbf{r}^{\prime \prime}, \mathbf{r}^{\prime}\right)=\mathbf{1} \delta\left(\mathbf{r}-\mathbf{r}^{\prime}\right), \quad \mathbf{r}, \mathbf{r}^{\prime} \in \delta V,
$$

i.e., the inversion is carried out on the subspace defined by the particle surface.

From the generating function Eq. (11) it is straightforward to calculate the mean flow profile as the functional derivative

$$
\left\langle u_{i}(\mathbf{r}, t)\right\rangle_{\mathbf{v}}=\left.\frac{\delta \ln Q}{\delta \phi_{i}(\mathbf{r}, t)}\right|_{\phi=0}
$$

with respect to the conjugate field. Using

$$
\frac{\delta b_{i}\left(\mathbf{r}^{\prime}, t^{\prime}\right)}{\delta \phi_{j}(\mathbf{r}, t)}=\left[\boldsymbol{\chi}_{0}\left(\mathbf{r}^{\prime}, \mathbf{r}\right)\right]_{i j} \delta\left(t-t^{\prime}\right)
$$

together with the symmetry property $\chi_{0}\left(\mathbf{r}^{\prime}, \mathbf{r}\right)=$ $\chi_{0}\left(\mathbf{r}, \mathbf{r}^{\prime}\right)$ we obtain

$$
\langle\mathbf{u}(\mathbf{r})\rangle_{\mathbf{v}}=\chi_{0}\left(\mathbf{r}, \mathbf{r}^{\prime}\right) \circ \chi_{\mathrm{s}}^{-1}\left(\mathbf{r}^{\prime}, \mathbf{r}^{\prime \prime}\right) \circ \mathbf{v} .
$$

For $\mathbf{r} \in \delta V$, we can use Eq. 12 to show that $\langle\mathbf{u}(\mathbf{r})\rangle_{\mathbf{v}}=$ $\mathbf{v}$ as required. Fig. 1(a) shows schematically how the imposed surface velocity generates the flow at $\mathbf{r}$ in the solvent through a concatenation of the tensors $\chi_{\mathrm{s}}^{-1}$ and $\chi_{0}$.

The presence of the solute not only causes a flow but also alters the solvent velocity fluctuations. The correla- 

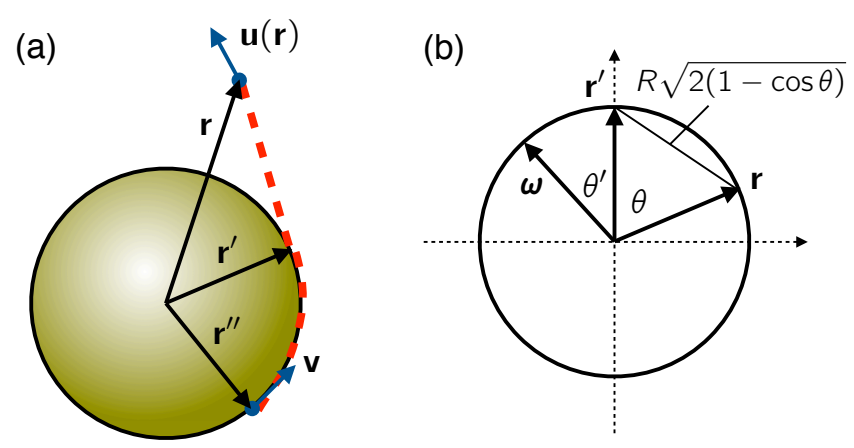

FIG. 1: (a) Schematic representation of Eq. (15): The flow $\mathbf{u}$ around the spherical particle at $\mathbf{r}$ is connected to the flow on the surface (given by the particle velocity $\mathbf{v}$ ) through a concatenation of the tensors $\chi_{\mathrm{s}}^{-1}$ and $\chi_{0}$. (b) Coordinate system and symbols used in the appendix: $\mathbf{r}$ and $\mathbf{r}^{\prime}$ are two points on the particle surface and $\boldsymbol{\omega}$ is the angular velocity.

tions are calculated as

$$
\left[\chi\left(\mathbf{r}, \mathbf{r}^{\prime}\right)\right]_{i j}=\left.\frac{\delta^{2} \ln Q}{\delta \phi_{i}(\mathbf{r}, t) \delta \phi_{j}\left(\mathbf{r}^{\prime}, t\right)}\right|_{\phi=0}
$$

and read

$$
\begin{aligned}
& \boldsymbol{\chi}\left(\mathbf{r}, \mathbf{r}^{\prime}\right)=\left\langle\delta \mathbf{u}(\mathbf{r}) \delta \mathbf{u}^{T}\left(\mathbf{r}^{\prime}\right)\right\rangle_{\mathbf{v}}= \\
& \boldsymbol{\chi}_{0}\left(\mathbf{r}, \mathbf{r}^{\prime}\right)-\boldsymbol{\chi}_{0}\left(\mathbf{r}, \mathbf{r}^{\prime \prime}\right) \circ \boldsymbol{\chi}_{\mathbf{s}}^{-1}\left(\mathbf{r}^{\prime \prime}, \mathbf{r}^{\prime \prime \prime}\right) \circ \boldsymbol{\chi}_{0}\left(\mathbf{r}^{\prime \prime \prime}, \mathbf{r}^{\prime}\right)
\end{aligned}
$$

for $\delta \mathbf{u}(\mathbf{r}) \equiv \mathbf{u}(\mathbf{r})-\langle\mathbf{u}(\mathbf{r})\rangle_{\mathbf{v}}$. For either $\mathbf{r} \in \delta V$ or $\mathbf{r}^{\prime} \in \delta V$, i.e., directly on the particle surface, the correlations vanish. Far away from the particle in the bulk they become those of the pure solvent, $\chi=\chi_{0}$.

\section{BROWNIAN MOTION}

Comparing the result Eq. (15) with the linear response relation Eq. (4), we see that it corresponds to a force density that is confined to the solute-solvent interface and given by

$$
\mathbf{f}(\mathbf{r})=2 T \boldsymbol{\chi}_{\mathrm{s}}^{-1}\left(\mathbf{r}, \mathbf{r}^{\prime}\right) \circ \mathbf{v}, \quad \mathbf{r} \in \delta V .
$$

The total force exerted by the particle follows from integrating this force density over the particle surface,

$$
\mathbf{F}=\mathbf{1} \circ \mathbf{f}=2 T\left(\mathbf{1} \circ \boldsymbol{\chi}_{\mathrm{s}}^{-1} \circ \mathbf{1}\right) \cdot \mathbf{v} \equiv \mathbf{\Gamma} \cdot \mathbf{v} .
$$

This is a linear relation between force and velocity, allowing us to identify $\boldsymbol{\Gamma}$ as the friction tensor. In appendix $\mathrm{A}$, $\boldsymbol{\Gamma}$ is calculated explicitly from Eq. (19) for a spherical particle.

So far, we have treated the particle velocity $\mathbf{v}$ as given. Inspecting Eq. (6), we see that for $\phi=0$ the generating function reduces to the path probability to observe spontaneous solvent velocity fluctuations that are compatible with the boundary condition of a uniform velocity on the solute surface. Hence, $P[\mathbf{v}(t)] \sim Q[\phi=0]$ is the path weight of a history of particle velocities with

$$
P[\mathbf{v}(t)] \equiv \exp \left\{-\frac{1}{4} \int \mathrm{d} t \mathbf{v}(t) \cdot \mathbf{D}^{-1} \cdot \mathbf{v}(t)\right\},
$$

which corresponds to the path weight of a free, overdamped Brownian particle. The diffusion tensor

$$
\mathbf{D}^{-1} \equiv 2\left(\mathbf{1} \circ \boldsymbol{\chi}_{\mathrm{s}}^{-1} \circ \mathbf{1}\right)=\boldsymbol{\Gamma} / T
$$

is given by the well-known Einstein relation connecting diffusion to friction through the fluid temperature.

\section{CONCLUSIONS}

The formalism introduced here is not restricted to spherical particles but can be used to obtain the friction tensors for arbitrarily shaped objects, e.g., colloidal clusters with complex shapes [13. Its practical use is somewhat hampered by the difficulties to determine $\boldsymbol{\chi}_{\mathrm{s}}^{-1}$ explicitly, which amounts to the inversion of $\chi_{0}$ on the two dimensional sub-manifold of the solute surface. Except for simple shapes (cf. appendix A) this has to be done numerically, possibly through an expansion of $\chi_{\mathrm{s}}$ into a suitable matrix basis. Rotational diffusion, and its coupling to translational diffusion, can be treated straightforwardly as demonstrated in appendix B Another advantage of the presented formalism is that we are not restricted to use the correlations Eq. (5) holding for an unbounded solvent. For example, one might calculate the pure solvent correlations $\chi_{0}\left(\mathbf{r}, \mathbf{r}^{\prime}\right)$ in some geometry numerically (e.g., using fluctuating lattice Boltzmann simulations [14) and use these correlations to obtain the diffusion coefficients of differently shaped objects moving in this geometry.

\section{Acknowledgments}

I thank David Chandler for introducing me to Gaussian field theory. I acknowledge financial support from the Alexander-von-Humboldt foundation.

\section{Appendix A: Friction tensor of a spherical particle}

To demonstrate the validity of the present formalism, we explicitly calculate the friction tensor for a spherical particle with radius $R$ via Eq. (19). We start by calculating the matrix

$$
\mathbf{X} \equiv \mathbf{1} \circ \boldsymbol{\chi}_{\mathrm{s}}=\int_{\delta V} \mathrm{~d}^{2} \mathbf{r} \boldsymbol{\chi}_{0}\left(\mathbf{r}-\mathbf{r}^{\prime}\right),
$$

which is the integral of the free correlations Eq. (5) over the surface of the sphere with $\mathbf{r}^{\prime}$ hold fixed. Without loss of generality, we place the particle center at the origin 
and use spherical coordinates with $\mathbf{r}^{\prime}$ pointing along the $z$-axis, see Fig. 1(b). The off-diagonal components vanish after integration over the azimuth angle. Substituting $z=\cos \theta$, the diagonal components read

$$
\begin{gathered}
X_{x x}=X_{y y}=\frac{T R}{2 \eta} \int_{-1}^{1} \mathrm{~d} z \frac{1}{\sqrt{2(1-z)}}\left[1+\frac{1-z^{2}}{4(1-z)}\right], \\
X_{z z}=\frac{T R}{2 \eta} \int_{-1}^{1} \mathrm{~d} z \frac{1+\frac{1}{2}(1-z)}{\sqrt{2(1-z)}}
\end{gathered}
$$

and therefore

$$
\mathbf{X}=\frac{4 T R}{3 \eta} \mathbf{1}
$$

As expected, this matrix does not depend on the vector $\mathbf{r}^{\prime}$ due to the spherical symmetry. Following Eq. (12), we find $\chi_{\mathrm{s}}^{-1} \circ \mathbf{1}=\mathbf{X}^{-1}$ and thus recover Stokes' expression

$$
\boldsymbol{\Gamma}=2 T\left(\mathbf{1} \circ \boldsymbol{\chi}_{\mathrm{s}}^{-1} \circ \mathbf{1}\right)=2 T 4 \pi R^{2} \mathbf{X}^{-1}=6 \pi \eta R \mathbf{1}
$$

for the friction tensor of a sphere.

\section{Appendix B: Rotational diffusion}

In order to include rotational diffusion, we have to modify the boundary condition. The fluid velocity on the surface now reads

$$
\mathbf{u}(\mathbf{r}, t)=\mathbf{v}(t)+\boldsymbol{\omega}(t) \times \mathbf{r}, \quad \mathbf{r} \in \delta V,
$$

where $\boldsymbol{\omega}=\omega \mathbf{e}$ is the angular velocity with speed $\omega$ about an axis given by the normalized vector e. Repeating the steps leading to Eq. (20), we obtain the path weight $P \sim e^{-A}$ with stochastic action

$$
A=\frac{1}{2} \int \mathrm{d} t[\mathbf{v}+\boldsymbol{\omega} \times \mathbf{r}] \circ \chi_{\mathrm{s}}^{-1}\left(\mathbf{r}, \mathbf{r}^{\prime}\right) \circ\left[\mathbf{v}+\boldsymbol{\omega} \times \mathbf{r}^{\prime}\right] .
$$

We now calculate the rotational diffusion coefficient for a spherical particle with radius $R$. We use a strategy similar to the previous section by multiplying Eq. (12) by the vector $\mathbf{e} \times \mathbf{r}$ from the left, and $\mathbf{e} \times \mathbf{r}^{\prime}$ from the right side followed by integrations,

$\int_{\delta V} \mathrm{~d}^{2} \mathbf{r} \mathbf{L}(\mathbf{r}) \cdot \mathbf{L}^{-1}(\mathbf{r})=\int_{\delta V} \mathrm{~d}^{2} \mathbf{r}(\mathbf{e} \times \mathbf{r}) \cdot(\mathbf{e} \times \mathbf{r})=\frac{8 \pi}{3} R^{4}$

Here, we have defined the vector field

$$
\mathbf{L}\left(\mathbf{r}^{\prime}\right) \equiv \int_{\delta V} \mathrm{~d}^{2} \mathbf{r} \chi_{0}\left(\mathbf{r}^{\prime}-\mathbf{r}\right) \cdot(\mathbf{e} \times \mathbf{r})=\frac{2}{3} \frac{T R}{\eta} \mathbf{e} \times \mathbf{r}^{\prime}
$$

It is convenient to calculate the integral using spherical coordinates as sketched in Fig. 1(b), where the $z$-axis points along $\mathbf{r}^{\prime}$. For the inverse field we thus find

$$
\mathbf{L}^{-1}\left(\mathbf{r}^{\prime}\right) \equiv \int_{\delta V} \mathrm{~d}^{2} \mathbf{r} \chi_{\mathrm{s}}^{-1}\left(\mathbf{r}^{\prime}, \mathbf{r}\right) \cdot(\mathbf{e} \times \mathbf{r})=\frac{\eta}{T R} \mathbf{e} \times \mathbf{r}^{\prime} .
$$

Using this vector, the stochastic action can be written

$$
A=\int \mathrm{d} t\left\{\frac{1}{4} \mathbf{v} \cdot \mathbf{D}^{-1} \cdot \mathbf{v}+\mathbf{v} \cdot\left(\mathbf{1} \circ \mathbf{L}^{-1}\right) \omega+\frac{\Gamma_{r}}{4 T} \omega^{2}\right\} .
$$

Clearly, $\mathbf{1} \circ \mathbf{L}^{-1}=0$, i.e., translational and rotational diffusion decouple. The rotational diffusion coefficient reads

$$
\Gamma_{r}=2 T \int_{\delta V} \mathrm{~d}^{2} \mathbf{r}(\mathbf{e} \times \mathbf{r}) \cdot \mathbf{L}^{-1}(\mathbf{r})=8 \pi \eta R^{3}
$$

as expected.
[1] A. Einstein, Ann. Phys. 17, 549 (1905).

[2] L. Landau and E. Lifshitz, Fluid mechanics, vol. 6 (Pergamon Press, Oxford, 1987), 2nd ed.

[3] E. H. Hauge and A. Martin-Löf, J. Stat. Phys. 7, 259 (1973).

[4] M. Schindler, Chem. Phys. 375, 327 (2010).

[5] D. Chandler, Phys. Rev. E 48, 2898 (1993).

[6] X. Song, D. Chandler, and R. A. Marcus, J. Phys. Chem. 100, 11954 (1996).

[7] K. Lum, D. Chandler, and J. D. Weeks, J. Phys. Chem. B 103, 4570 (1999).

[8] A. Widom, Phys. Rev. A 3, 1394 (1971).

[9] T. Franosch, M. Grimm, M. Belushkin, F. M. Mor,
G. Foffi, L. Forro, and S. Jeney, Nature 478, 85 (2011).

[10] F. Detcheverry and L. Bocquet, Phys. Rev. Lett. 109, 024501 (2012).

[11] R. Kubo, M. Toda, and N. Hashitsume, Statistical Physics II (Springer-Verlag, Berlin, 1991), 2nd ed.

[12] J. K. G. Dhont, An Introduction to Dynamics of Colloids (Elsevier, Amsterdam, 1996).

[13] D. J. Kraft, R. Wittkowski, B. ten Hagen, K. V. Edmond, D. J. Pine, and H. Löwen, arXiv:1305.1253 (2013).

[14] R. Adhikari, K. Stratford, M. E. Cates, and A. J. Wagner, Europhys. Lett. 71, 473 (2005). 